\title{
Activity of Tedizolid in Methicillin-Resistant Staphylococcus aureus Experimental Foreign Body-Associated Osteomyelitis
}

\author{
Kyung-Hwa Park, ${ }^{\text {a,d }}$ Kerryl E. Greenwood-Quaintance, ${ }^{a}$ Jayawant Mandrekar, ${ }^{\text {b }}$ Robin Patel $^{\text {a,c }}$ \\ Division of Clinical Microbiology, Department of Laboratory Medicine and Pathology, ${ }^{a}$ Division of Biomedical Statistics and Informatics, ${ }^{b}$ and Division of Infectious \\ Diseases, Department of Medicine, ${ }^{c}$ Mayo Clinic, Rochester, Minnesota, USA; Department of Infectious Diseases, Chonnam National University Medical School, Gwangju, \\ South Korea ${ }^{\mathrm{d}}$
}

We compared tedizolid alone and tedizolid with rifampin to rifampin and vancomycin plus rifampin in a rat model of methicillin-resistant Staphylococcus aureus (MRSA) foreign body-associated osteomyelitis. The study strain was a prosthetic joint infection-associated isolate. Steady-state pharmacokinetics for intraperitoneal administration of tedizolid, vancomycin, and rifampin were determined in uninfected rats. MRSA was inoculated into the proximal tibia, and a wire was implanted. Four weeks later, the rats were treated intraperitoneally for 21 days with tedizolid $(n=14)$, tedizolid plus rifampin $(n=11)$, rifampin $(n=16)$, or vancomycin plus rifampin $(n=13)$. Seventeen rats received no treatment. After treatment, quantitative bone cultures were performed. Blood was obtained for determination of drug trough concentrations in the tedizolid and tedizolid plus rifampin groups. The mean peak plasma concentration and mean area under the concentration-time curve from time zero to $24 \mathrm{~h}$ for tedizolid were $12 \mu \mathrm{g} / \mathrm{ml}$ and $60 \mu \mathrm{g} \cdot \mathrm{h} / \mathrm{ml}$, respectively. The bacterial loads in all treatment groups were significantly lower than those in the control group; those in the tedizolid- plus rifampin-treated animals were not significantly different from those in the vancomycin- plus rifampin-treated animals. The range of mean plasma trough concentrations in the tedizolid group was 0.44 to 0.73 $\mu \mathrm{g} / \mathrm{ml}$. Although neither tedizolid nor vancomycin resistance was detected in isolates recovered from bones, rifampin resistance was detected in 10 animals (63\%) in the rifampin group, 8 animals $(73 \%)$ in the tedizolid plus rifampin group, and a single animal $(8 \%)$ in the vancomycin plus rifampin group. Tedizolid alone or tedizolid combined with rifampin was active in a rat model of MRSA foreign body-associated osteomyelitis. The emergence of rifampin resistance was noted in animals receiving tedizolid plus rifampin.

O ver half of chronic device-associated infections are caused by staphylococci, with Staphylococcus aureus and Staphylococcus epidermidis being the most common (1). In the United States, up to $47 \%$ of $S$. aureus strains and $86 \%$ of coagulase-negative staphylococcal strains causing prosthetic joint infection are methicillin resistant (2). Moreover, the growth of bacteria in biofilms challenges treatment of orthopedic foreign body-associated infections. Within biofilms, an altered microenvironment compromises antimicrobial action as a result of the presence of persister cells, decreased antimicrobial activity against stationary-growthphase bacteria, modification of some antimicrobial agents by inactivating enzymes, and, to some extent and with certain antimicrobial agents, decreased penetration (3).

Management of orthopedic device-associated infections typically involves antimicrobial treatment in combination with surgical intervention. Options for treating methicillin-resistant staphylococci in device-associated infections are especially limited (4). While vancomycin is considered the "gold standard" therapy, its clinical activity against staphylococci is debated due to MIC values that may approach its susceptible breakpoint, challenges achieving adequate serum levels, heteroresistance, tolerance, and side effects, compounded by the occasional presence of vancomycinintermediate staphylococci (5). Linezolid is a consideration, but it is bacteriostatic, may have variable interindividual pharmacokinetics, may have monoamine oxidase inhibitor interactions, and may cause myelosuppression, lactic acidosis, and hepatic dysfunction $(6,7)$. Recently, linezolid- and daptomycin-resistant Grampositive cocci have been reported $(8,9)$.

Tedizolid is an expanded-spectrum oxazolidinone approved by the United States Food and Drug Administration for the treat- ment of acute bacterial skin and skin structure infections; it has greater potency against methicillin-resistant Staphylococcus aureus (MRSA) than does linezolid, and data from comparative clinical trials suggest that it may have less toxicity, including fewer toxic effects resulting in thrombocytopenia and neuropathy, and fewer monoamine oxidase inhibitor interactions than linezolid (10-12). Moreover, once-daily dosing is possible. Tedizolid has not been studied in an animal model of foreign body-associated osteomyelitis.

In the study described here, tedizolid with and without rifampin was compared to rifampin monotherapy and vancomycin combined with rifampin in a rat model of foreign body-associated osteomyelitis established with a prosthetic joint infection-associated MRSA strain.

\section{MATERIALS AND METHODS}

Microorganism and antimicrobial agents. MRSA IDRL-6169, originally recovered from a patient with a prosthetic joint infection, was studied. Vancomycin was obtained from Hospira Inc. (Lake Forest, IL). Tedizolid

Received 10 June 2016 Returned for modification 6 July 2016 Accepted 12 August 2016

Accepted manuscript posted online 22 August 2016

Citation Park K-H, Greenwood-Quaintance KE, Mandrekar J, Patel R. 2016. Activity of tedizolid in methicillin-resistant Staphylococcus aureus experimental foreign body-associated osteomyelitis. Antimicrob Agents Chemother 60:6568-6572. doi:10.1128/AAC.01248-16.

Address correspondence to Robin Patel, patel.robin@mayo.edu.

Copyright @ 2016, American Society for Microbiology. All Rights Reserved. 
was obtained from Merck \& Co., Inc. (Whitehouse Station, NJ). Rifampin was obtained from Akorn Inc. (Lake Forest, IL). The MIC of each antimicrobial agent was determined by broth microdilution using an inoculum of $5 \times 10^{5} \mathrm{CFU} / \mathrm{ml}$ according to Clinical and Laboratory Standards Institute (CLSI) guidelines (13). For biofilm assays, the minimum biofilm inhibitory concentration (MBIC) and the minimum biofilm bactericidal concentration (MBBC) were determined as previously described (14). S. aureus ATCC 29213 was used for quality control.

Pharmacokinetic studies. Drug doses were selected as follows. The rifampin dose was selected to simulate the concentration above the MIC observed in humans. The vancomycin dose was selected to simulate the time above the MIC observed in humans. The tedizolid dose was selected to simulate the free area under the concentration-time curve (AUC) observed in humans after administration of $200 \mathrm{mg}$. All pharmacokinetic profiles were assessed in five healthy male Wistar rats after three doses (steady state) prior to implementation of the treatment model. The concentrations of rifampin and vancomycin after intraperitoneal administration were previously determined by serum bioassay $(15,16)$. The concentration of tedizolid to be used in the study, $30 \mathrm{mg} / \mathrm{kg}$ of body weight once daily (tedizolid phosphate for injection), administered intraperitoneally to 10 rats, was determined by measuring the plasma concentrations at 0.5 , $1,2,4,6$, and $12 \mathrm{~h}$ after the third daily dose. Blood $(\sim 500 \mu \mathrm{l})$ was collected from a central line from five animals at each time point, with the same five animals being resampled at alternating time points. Blood was placed in Greiner 0.5-ml K3 EDTA-containing tubes (Instech Laboratories, Inc., Plymouth Meeting, PA), and the plasma was separated by centrifugation. Plasma samples were stored at $-80^{\circ} \mathrm{C}$; the plasma concentrations of tedizolid were analyzed at Charles River Laboratories (Wilmington, MA) by high-performance liquid chromatography (HPLC) using a UV detector (PerkinElmer 200 series micropumps; PerkinElmer, Waltham, MA, USA) and a mass spectrometer (API 4000; AB Sciex, Framingham, MA, USA). The conditions for HPLC-tandem mass spectrometry for plasma tedizolid analysis were as follows. An Xbridge $\mathrm{C}_{18}$ (30 by $2.1 \mathrm{~mm}$; Waters, Milford, MA, USA) capillary column with a mobile phase that consisted of $0.1 \%$ formic acid in water and $0.1 \%$ formic acid in acetonitrile was used. The analytical process was validated for tedizolid concentrations ranging from 2.5 to $1,000 \mathrm{ng} / \mathrm{ml}$.

Animal model. The experimental model described was developed and performed in accordance with the guidelines of the Institutional Animal Care and Use Committee of the Mayo Clinic. Experimental foreign bodyassociated osteomyelitis was established in male Wistar rats weighing 250 to $350 \mathrm{~g}$ as previously described (15). Briefly, general anesthesia was induced by intraperitoneal administration of ketamine $(60 \mathrm{mg} / \mathrm{kg})$ and $\mathrm{xy}-$ lazine $(6 \mathrm{mg} / \mathrm{kg})$. After surgical anesthesia, the left legs of the rats were shaved, the skin was disinfected, the proximal third of the left tibia was surgically exposed, and a $1.5-\mathrm{mm}$ hole was drilled into the medullary cavity. Fifty microliters of a $10^{6}$-CFU suspension of the MRSA isolate was injected into the bone. Subsequently, a $5-\mathrm{mm}$ by $1-\mathrm{mm}$ stainless steel wire (Zimmer, Warsaw, IN) was implanted into the bone. The hole was covered with dental gypsum. The fascia and skin were closed with sutures, and the wound was sprayed with antiseptic. An extended-release suspension of buprenorphine $(0.05 \mathrm{mg} / \mathrm{kg}$, active for 3 days $)$ was administered once subcutaneously for analgesia.

Four weeks after establishing infection, treatment was initiated. In total, 82 animals (weight, 332 to $434 \mathrm{~g}$ ) were treated in one of five study arms: no treatment $(n=18)$, treatment with tedizolid alone $(n=16)$, treatment with tedizolid plus rifampin $(n=16)$, treatment with rifampin alone $(n=16)$, or treatment with vancomycin plus rifampin $(n=16)$. The data for rifampin monotherapy and vancomycin plus rifampin combination therapy and the data for 13 untreated rats have been published previously (15). Two animals each in the tedizolid and tedizolid plus rifampin groups died following surgery. Tedizolid was administered at 30 $\mathrm{mg} / \mathrm{kg}$ intraperitoneally once daily, rifampin was administered at 25 $\mathrm{mg} / \mathrm{kg}$ intraperitoneally twice daily, and vancomycin was administered at $50 \mathrm{mg} / \mathrm{kg}$ intraperitoneally twice daily. Treatment was administered for 21 days. Animals not surviving 21 days of antimicrobial treatment were not further studied. During treatment, there were three deaths, and all were in the vancomycin plus rifampin group. One animal in the no-treatment group and three in the tedizolid plus rifampin group in which no wire was found in the tibia at the time of sacrifice (presumably due to migration of the wire) were excluded from further analyses.

At $24 \mathrm{~h}$ after completion of tedizolid therapy and at $12 \mathrm{~h}$ after completion of the other antimicrobial therapies, the rats were sacrificed using $\mathrm{CO}_{2}$ and the left tibia was aseptically removed. The tibia was frozen to $-80^{\circ} \mathrm{C}$. Bone within $5 \mathrm{~mm}$ of the implanted stainless steel wire from each animal was weighed and pulverized. Pulverized bone and wire were separately suspended in $2 \mathrm{ml}$ of Trypticase soy broth, vortexed for $30 \mathrm{~s}$, and sonicated at $40 \mathrm{kHz}$ for $5 \mathrm{~min}$. Quantitative bone cultures were performed as previously described (15), with results expressed as the $\log _{10}$ number of CFU per gram of bone. If there was no growth in the quantitative cultures, qualitative cultures were performed by adding $8 \mathrm{ml}$ of sterile Trypticase soy broth to the remaining $2 \mathrm{ml}$ broth containing pulverized bone and incubating the mixture for $48 \mathrm{~h}$ at $37^{\circ} \mathrm{C}$. For broth with evidence of growth, subcultures were performed to confirm the presence of MRSA. The wire was qualitatively cultured as described above.

Plasma tedizolid and rifampin trough concentrations. Blood was obtained from the tail vein of seven animals in each of the tedizolid and tedizolid plus rifampin treatment groups after 1 week of treatment and from the remaining seven animals in each group after 2 weeks of treatment for determination of trough concentrations. Blood was also obtained via cardiac puncture at the time of sacrifice (i.e., after 3 weeks of postinfection treatment). Plasma samples were stored at $-80^{\circ} \mathrm{C}$ for determination of tedizolid and rifampin trough concentrations, both of which were determined by HPLC as described above (with the rifampin concentrations being determined by the standard HPLC assay at Charles River Laboratories).

Antimicrobial resistance studies. The MIC of tedizolid, rifampin, or vancomycin for MRSA isolates recovered from bone after treatment was determined using three colonies from quantitative culture plates for each animal (unless less than three colonies were available).

Statistical methods. Descriptive statistics for the $\log _{10}$ number of CFU of MRSA per gram of bone were summarized as medians and interquartile ranges (IQRs). For statistical purposes, we considered the absence of growth to be $0.1 \log _{10} \mathrm{CFU} / \mathrm{g}$ of bone. We considered growth in qualitative broth culture only (but not on quantitative cultures plates) to be $0.5 \log _{10}$ $\mathrm{CFU} / \mathrm{g}$ of bone. Differences in the median $\log _{10}$ number of CFU/g of bone among the five groups were compared using the Kruskal-Wallis test. Further, pairwise comparisons of the median $\log _{10} \mathrm{CFU} / \mathrm{g}$ of bone between the five groups (i.e., the control group and the four treatment groups) were made using the Wilcoxon rank sum test. On the basis of the Bonferroni correction for adjustment due to multiple comparisons for bone, $P$ values of less than 0.005 were considered statistically significant. Differences in the qualitative broth cultures of wire between the untreated and treated groups were compared using Fisher's exact test. Drug concentrations were compared using the Student $t$ test. For comparison of the emergence of rifampin resistance among the treatment groups, Fisher's exact test was used. All tests were two-sided, and a $P$ value of $<0.05$ was considered to indicate statistical significance, except for bone, as noted above. Analysis was performed using SAS software (version 9; SAS Institute, Inc., Cary, NC).

\section{RESULTS}

MICs, MBICs, and MBBCs. The MICs of each of the antimicrobials for the MRSA study isolate were as follows: oxacillin, $>128$ $\mu \mathrm{g} / \mathrm{ml}$; vancomycin, $1 \mu \mathrm{g} / \mathrm{ml}$; rifampin, $\leq 0.015 \mu \mathrm{g} / \mathrm{ml}$; and tedizolid, $0.5 \mu \mathrm{g} / \mathrm{ml}$. The MBICs for the isolate were $0.5 \mu \mathrm{g} / \mathrm{ml}$ for vancomycin, $0.002 \mu \mathrm{g} / \mathrm{ml}$ for rifampin, and $0.5 \mu \mathrm{g} / \mathrm{ml}$ for tedizolid. The MBBCs for the isolate were $>128 \mu \mathrm{g} / \mathrm{ml}$ for vancomycin, $2 \mu \mathrm{g} / \mathrm{ml}$ for rifampin, and $>32 \mu \mathrm{g} / \mathrm{ml}$ for tedizolid.

Pharmacokinetics in uninfected animals. The pharmacoki- 
TABLE 1 Pharmacokinetic parameters for the study antimicrobial agents ${ }^{a}$

\begin{tabular}{lllllllll}
\hline & \multicolumn{2}{l}{ Mean \pm SD drug concn $(\mu \mathrm{g} / \mathrm{ml})$} & & \\
\cline { 2 - 6 } Antimicrobial (dosing) & $0.5 \mathrm{~h}$ & $1 \mathrm{~h}$ & $2 \mathrm{~h}$ & $4 \mathrm{~h}$ & $6 \mathrm{~h}$ & $12 \mathrm{~h}$ & $C_{\max }(\mu \mathrm{g} / \mathrm{ml})$ & $\begin{array}{c}\mathrm{AUC}_{0-24} \\
(\mu \mathrm{g} \cdot \mathrm{h} / \mathrm{ml})\end{array}$ \\
\hline Vancomycin (50 mg/kg i.p. twice daily) & $47 \pm 8.2$ & $26 \pm 6.2$ & $14 \pm 4.4$ & $8.2 \pm 1.4$ & $7.4 \pm 2.4$ & $<2.0$ & 12 \\
Rifampin (25 mg/kg i.p. twice daily) & $28 \pm 6.6$ & $19 \pm 1.9$ & $14 \pm 7.9$ & $13 \pm 1.9$ & $12 \pm 2.9$ & $8.9 \pm 0.8$ & 47 \\
Tedizolid (30 mg/kg i.p. once daily) & $12 \pm 5.3$ & $7.1 \pm 0.9$ & $11 \pm 4.3$ & $3.3 \pm 0.6$ & $3.1 \pm 0.6$ & $1.6 \pm 0.2$ & 28 & 332 \\
\hline
\end{tabular}

${ }^{a} C_{\max }$, maximum concentration of drug in plasma; i.p., intraperitoneal.

netics of vancomycin, rifampin, and tedizolid are shown in Table 1. The data for rifampin (15) and vancomycin (16) were obtained from previous studies. Drug concentrations after $12 \mathrm{~h}$ of administration of each antimicrobial were as follows: vancomycin, $<2.0$ $\mu \mathrm{g} / \mathrm{ml}$; rifampin, $8.9 \mu \mathrm{g} / \mathrm{ml}$; and tedizolid, $1.6 \mu \mathrm{g} / \mathrm{ml}$. The AUC from time zero to $24 \mathrm{~h}\left(\mathrm{AUC}_{0-24}\right)$ was $60 \mu \mathrm{g} \cdot \mathrm{h} / \mathrm{ml}$ for tedizolid administered intraperitoneally at $30 \mathrm{mg} / \mathrm{kg}$ once daily. The total tedizolid AUC was twice that at steady state for the human intravenous dose of $200 \mathrm{mg}$ once daily (17). The $\mathrm{AUC}_{0-24}$ for vancomycin administered intraperitoneally at $50 \mathrm{mg} / \mathrm{kg}$ twice daily was $264 \mu \mathrm{g} \cdot \mathrm{h} / \mathrm{ml}$, and that for rifampin administered intraperitoneally at $25 \mathrm{mg} / \mathrm{kg}$ twice daily was $332 \mu \mathrm{g} \cdot \mathrm{h} / \mathrm{ml}$.

Experimental rat model. The results of quantitative cultures of bone after treatment are summarized in Fig. 1. MRSA was cultured from all harvested bones in the control group at a median count of $5.76 \log _{10} \mathrm{CFU} / \mathrm{g}$ of bone (interquartile range [IQR], 5.37 to $6.65 \log _{10} \mathrm{CFU} / \mathrm{g}$ of bone). After 3 weeks of treatment, the median count of MRSA was $3.86 \log _{10} \mathrm{CFU} / \mathrm{g}$ of bone (IQR, 2.88 to $4.83 \log _{10} \mathrm{CFU} / \mathrm{g}$ of bone) in the tedizolid group and $2.95 \log _{10}$ CFU/g of bone (IQR, 0.50 to $4.0 \log _{10} \mathrm{CFU} / \mathrm{g}$ of bone) in the tedizolid plus rifampin group. The median count of MRSA was $1.35 \log _{10} \mathrm{CFU} / \mathrm{g}$ of bone (IQR, 0.10 to $3.34 \log _{10} \mathrm{CFU} / \mathrm{g}$ of bone) in the rifampin group and $0.50 \log _{10} \mathrm{CFU} / \mathrm{g}$ of bone (IQR, 0.10 to $2.66 \log _{10} \mathrm{CFU} / \mathrm{g}$ of bone) in the vancomycin plus rifampin group.
Differences in colony counts compared to the colony count for the control group were significant for all treatment groups $(P \leq$ $0.0001)$. There was no significant difference between the tedizolid and tedizolid plus rifampin groups $(P=0.07)$, between the rifampin and vancomycin plus rifampin groups $(P=0.70)$, or between the tedizolid plus rifampin and vancomycin plus rifampin groups $(P=0.16)$. However, there was a significant difference between the tedizolid alone and vancomycin plus rifampin groups $(P=$ 0.0009).

Qualitative cultures of the stainless steel wires were also obtained. MRSA was cultured from stainless steel wires from all control animals except one. Results of qualitative cultures were as follows (the $P$ values shown are with reference to the results for the control animals). There was growth in 1/16 animals in the rifampin group $(P<0.001), 8 / 14$ animals in the tedizolid group $(P=$ $0.03), 6 / 11$ animals in the tedizolid plus rifampin group $(P=$ 0.02 ), and $0 / 13$ animals in the vancomycin plus rifampin group $(P<0.001)$.

Plasma tedizolid and rifampin trough concentrations in infected animals. The mean \pm standard deviation (SD) plasma trough concentration of tedizolid was $0.55 \pm 0.38 \mu \mathrm{g} / \mathrm{ml}$ after 1 week of treatment, $0.73 \pm 0.59 \mu \mathrm{g} / \mathrm{ml}$ after 2 weeks of treatment, and $0.44 \pm 0.23 \mu \mathrm{g} / \mathrm{ml}$ after 3 weeks of treatment in the tedizolid monotherapy group (Table 2 ). The mean \pm SD plasma trough

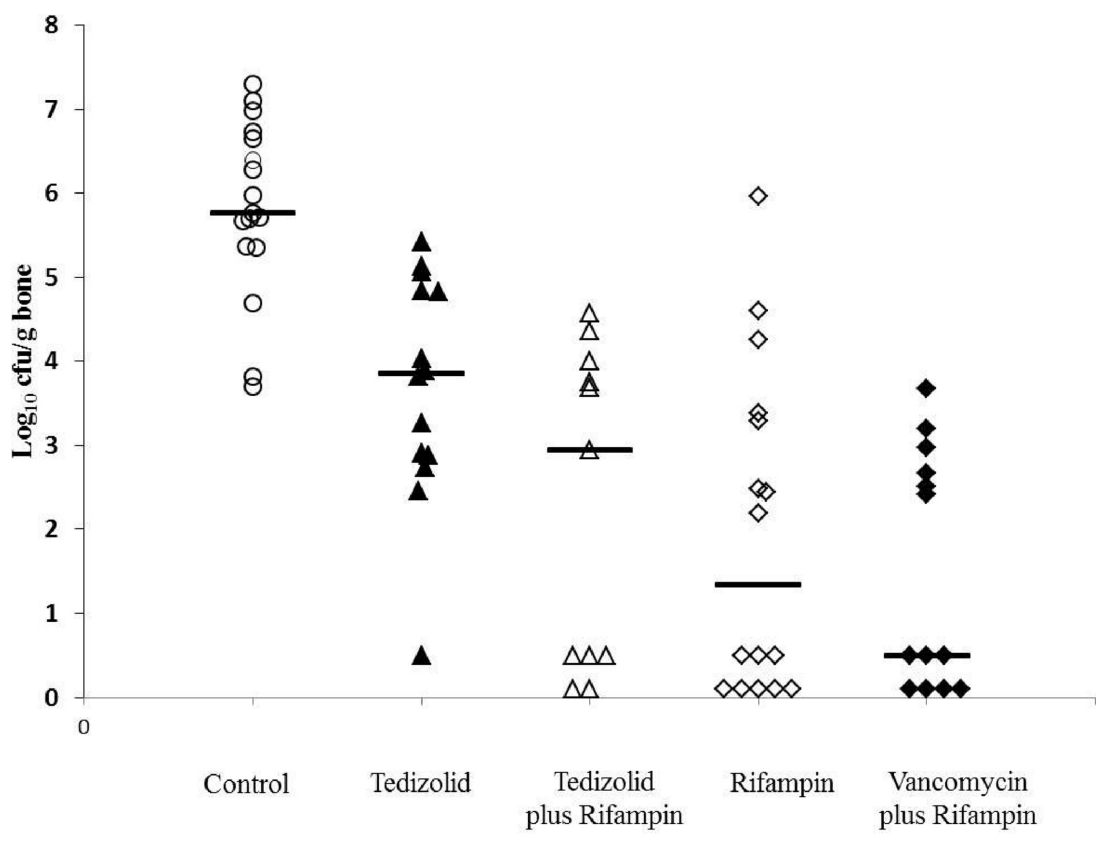

FIG 1 Results of quantitative bone culture. The median value (bars) and individual results (individual symbols) are shown for each study group. Differences between the control group and each treatment group were statistically significant $(P \leq 0.0001)$. 
TABLE 2 Plasma trough concentrations of tedizolid and rifampin

\begin{tabular}{|c|c|c|c|c|c|}
\hline \multirow[b]{2}{*}{ Treatment group } & \multirow[b]{2}{*}{$\begin{array}{l}\text { Treatment } \\
\text { duration (wk) }\end{array}$} & \multicolumn{2}{|l|}{ Tedizolid } & \multicolumn{2}{|l|}{ Rifampin } \\
\hline & & $\begin{array}{l}\text { Mean } \pm \text { SD trough } \\
\text { concn }(\mu \mathrm{g} / \mathrm{ml})\end{array}$ & $P$ value & $\begin{array}{l}\text { Mean } \pm \text { SD trough } \\
\text { concn }(\mu \mathrm{g} / \mathrm{ml})\end{array}$ & $P$ value \\
\hline \multirow[t]{3}{*}{ Tedizolid } & 1 & $0.55 \pm 0.38$ & 0.75 ( 1 wk vs 2 wk) & $\mathrm{NA}^{a}$ & \\
\hline & 2 & $0.73 \pm 0.59$ & 0.37 ( 2 wk vs 3 wk) & NA & \\
\hline & 3 & $0.44 \pm 0.23$ & 0.81 ( 1 wk vs 3 wk) & NA & \\
\hline \multirow[t]{3}{*}{ Tedizolid plus rifampin } & 1 & $0.77 \pm 0.68$ & 0.04 ( 1 wk vs 2 wk) & $6.59 \pm 3.26$ & 0.57 ( 1 wk vs 2 wk) \\
\hline & 2 & $1.77 \pm 0.77$ & 0.29 ( 2 wk vs 3 wk) & $6.17 \pm 3.14$ & 0.01 ( 2 wk vs 3 wk) \\
\hline & 3 & $1.07 \pm 1.01$ & 0.94 ( 1 wk vs 3 wk) & $1.97 \pm 1.11$ & 0.03 ( 1 wk vs 3 wk) \\
\hline
\end{tabular}

${ }^{a}$ NA, not applicable.

concentration of tedizolid was $0.77 \pm 0.68 \mu \mathrm{g} / \mathrm{ml}$ after 1 week of treatment, $1.77 \pm 0.77 \mu \mathrm{g} / \mathrm{ml}$ after 2 weeks of treatment, and $1.07 \pm 1.01 \mu \mathrm{g} / \mathrm{ml}$ after 3 weeks of treatment in the tedizolid plus rifampin combination therapy group. After 2 weeks of treatment, the mean plasma trough concentration of tedizolid was higher in the tedizolid plus rifampin combination therapy group than the tedizolid monotherapy group $(P=0.04)$, but the values were similar at 1 and 3 weeks. In the tedizolid plus rifampin group, a trend according to time of treatment showed differences in tedizolid concentrations between 1 and 2 weeks $(P=0.04)$. In the tedizolid plus rifampin group, the mean plasma concentration of rifampin was lower at 3 weeks $(1.97 \pm 1.11)$ than at 1 or 2 weeks $(6.59 \pm$ 3.26 and $6.17 \pm 3.14 \mu \mathrm{g} / \mathrm{ml}$, respectively) of treatment $(P=0.03$, 1 week versus 3 weeks; $P=0.01,2$ weeks versus 3 weeks).

Emergence of resistance. Emergence of resistance (i.e., a posttreatment rifampin $\mathrm{MIC}$ of $>4 \mu \mathrm{g} / \mathrm{ml}$ ) was detected in 10 animals $(63 \%)$ in the rifampin group, 8 animals $(73 \%)$ in the tedizolid plus rifampin group, and 1 animal (8\%) in the vancomycin plus rifampin group. The differences between the rifampin monotherapy and vancomycin plus rifampin groups $(P=0.006)$ and between the tedizolid plus rifampin and vancomycin plus rifampin groups $(P=0.002)$ were statistically significant, but there was no difference between the rifampin monotherapy and tedizolid plus rifampin groups $(P=0.69)$. The findings remained significant, in spite of adjustment for multiple comparisons. There was no emergence of resistance to tedizolid or vancomycin in the respective treatment groups.

\section{DISCUSSION}

In the present study, we demonstrated that treatment with tedizolid alone or the combination of tedizolid plus rifampin or vancomycin plus rifampin is more effective than no treatment in an animal model of MRSA foreign body-associated osteomyelitis in the context of retention of the infected foreign body.

For our rat studies, we selected a tedizolid dose of $30 \mathrm{mg} / \mathrm{kg}$, which achieves an AUC higher than that achieved in humans because the level of tedizolid protein binding in rats $(>90 \%)$ is higher than that in humans (80\%) (18). As such, the rat free AUC (fAUC) is actually below the human free AUC. A dose fractionation study has shown that the free-drug AUC over $24 \mathrm{~h}$ at steady state $\left[\mathrm{AUC}_{\mathrm{ss}(0-24)}\right]$ divided by the MIC (i.e., the fAUC/MIC ratio) is the pharmacokinetic/pharmacodynamic parameter that best correlates with efficacy against MRSA (19).

There were three deaths in the vancomycin plus rifampin group and no deaths in the tedizolid plus rifampin group. The cause of death in the vancomycin plus rifampin group is not known. In the tedizolid plus rifampin treatment group, the trough concentrations of tedizolid at $24 \mathrm{~h}$ posttreatment (range, 0.77 to $1.77 \mu \mathrm{g} / \mathrm{ml}$ ) were similar to those in the healthy animals in the pharmacokinetic study. The values obtained by serum liver and kidney chemistry tests in the no-treatment, tedizolid, and tedizolid plus rifampin groups at the time of sacrifice were within the normal range (data not shown). Linezolid is known to cause thrombocytopenia, particularly when it is used for more than 2 weeks; maintenance of a serum linezolid trough concentration of between 2 and $7 \mu \mathrm{g} / \mathrm{ml}$ has been suggested as a step for improving safety outcomes (20). In preclinical studies, there was myelosuppression in tedizolid-treated rats and dogs with increasing drug exposures, similar to the findings for linezolid $(21,22)$. We did not observe any overt toxicity with tedizolid plus rifampin administered for 21 days, although the purpose of our study was not to specifically assess toxicity.

We previously observed that vancomycin or linezolid monotherapy did not reduce bacterial counts in the rat foreign bodyassociated osteomyelitis model with the same protocol (23), but in the present study, tedizolid monotherapy did reduce bacterial counts compared to the counts achieved with no treatment. The in vitro potency of tedizolid is 2- to 8-fold more than that of vancomycin against staphylococci, and its prolonged subinhibitory MIC and postantibiotic effect against staphylococci are greater than those of linezolid (24). Recently, Chan et al. reported that tedizolid and vancomycin had comparable activity in a rabbit model of low-inoculum MRSA endocarditis (25). To our knowledge, this is the first study of combination treatment with tedizolid plus rifampin in an animal model of device-associated infection.

There was no emergence of resistance for either tedizolid or vancomycin. Rifampin monotherapy was associated with a significant emergence of resistance, as expected (26). However, rifampin resistance was also detected in the vancomycin plus rifampin and tedizolid plus rifampin combination treatment groups. Our pharmacokinetic studies showed that the tedizolid, vancomycin, and rifampin levels obtained a half hour after injection were above the peak levels considered therapeutic in humans. Rifampin trough levels were detectable. Nonetheless, in the tedizolid plus rifampin combination group, the rifampin trough concentrations after 3 weeks of treatment were lower than those after 1 week or 2 weeks of treatment. Unfortunately, rifampin trough levels were not measured in the rifampin monotherapy or vancomycin plus rifampin group. However, the low trough level of rifampin in the tedizolid plus rifampin combination group may have contributed to the emergence of rifampin resistance. Tedizolid is not known to 
affect cytochrome P450 enzymes, and no potential drug interactions with tedizolid have been identified by in vitro cytochrome P450 enzyme inhibition or induction studies (17). The clinical relevance of this observation is unknown.

The limitations of the present study include the facts that only one bacterial strain was tested, that rifampin trough levels were not checked in all treatment groups, and that there were some animal and wire losses.

In conclusion, tedizolid monotherapy or tedizolid plus rifampin combination therapy is active against MRSA in a rat model of foreign body-associated osteomyelitis; however, the emergence of rifampin resistance was noted in animals receiving tedizolid plus rifampin.

\section{ACKNOWLEDGMENT}

This study was supported by Merck.

\section{FUNDING INFORMATION}

This work, including the efforts of Kyung-Hwa Park, Kerryl E. Greenwood-Quaintance, and Robin Patel, was funded by Merck (Merck \& Co., Inc.).

\section{REFERENCES}

1. Del Pozo JL, Patel R. 2009. Clinical practice. Infection associated with prosthetic joints. N Engl J Med 361:787-794.

2. Garvin KL, Hinrichs SH, Urban JA. 1999. Emerging antibiotic-resistant bacteria. Their treatment in total joint arthroplasty. Clin Orthop Relat Res 1999:110-123.

3. Del Pozo JL, Patel R. 2007. The challenge of treating biofilm-associated bacterial infections. Clin Pharmacol Ther 82:204-209. http://dx.doi.org /10.1038/sj.clpt.6100247.

4. Osmon DR, Berbari EF, Berendt AR, Lew D, Zimmerli W, Steckelberg JM, Rao N, Hanssen A, Wilson WR. 2013. Diagnosis and management of prosthetic joint infection: clinical practice guidelines by the Infectious Diseases Society of America. Clin Infect Dis 56:e1-e25. http://dx.doi.org /10.1093/cid/cis803.

5. Holmes NE, Johnson PD, Howden BP. 2012. Relationship between vancomycin-resistant Staphylococcus aureus, vancomycin-intermediate $S$. aureus, high vancomycin MIC, and outcome in serious $S$. aureus infections. J Clin Microbiol 50:2548-2552. http://dx.doi.org/10.1128/JCM $.00775-12$.

6. Gould FK. 2011. Linezolid: safety and efficacy in special populations. J Antimicrob Chemother 66(Suppl 4):iv3-iv6. http://dx.doi.org/10.1093 /jac/dkr071.

7. Dehghanyar P, Burger C, Zeitlinger M, Islinger F, Kovar F, Muller M, Kloft C, Joukhadar C. 2005. Penetration of linezolid into soft tissues of healthy volunteers after single and multiple doses. Antimicrob Agents Chemother 49:2367-2371. http://dx.doi.org/10.1128 /AAC.49.6.2367-2371.2005.

8. Silva-Del Toro SL, Greenwood-Quaintance KE, Patel R. 2016. In vitro activity of tedizolid against linezolid-resistant staphylococci and enterococci. Diagn Microbiol Infect Dis 85:102-104. http://dx.doi.org/10.1016/j .diagmicrobio.2016.02.008.

9. Mishra NN, Yang SJ, Chen L, Muller C, Saleh-Mghir A, Kuhn S, Peschel A, Yeaman MR, Nast CC, Kreiswirth BN, Crémieux AC, Bayer AS. 2013. Emergence of daptomycin resistance in daptomycin-naive rabbits with methicillin-resistant Staphylococcus aureus prosthetic joint infection is associated with resistance to host defense cationic peptides and $\mathrm{mprF}$ polymorphisms. PLoS One 8:e71151. http://dx.doi.org/10.1371/journal .pone.0071151.

10. Thomson KS, Goering RV. 2013. Activity of tedizolid (TR-700) against well-characterized MRSA strains of diverse epidemiological origin. Antimicrob Agents Chemother 57:2892-2895. http://dx.doi.org/10.1128/AAC .00274-13.

11. Rybak JM, Marx K, Martin CA. 2014. Early experience with tedizolid: clinical efficacy, pharmacodynamics, and resistance. Pharmacotherapy 34:1198-1208. http://dx.doi.org/10.1002/phar.1491.

12. Lodise TP, Bidell MR, Flanagan SD, Zasowski EJ, Minassian SL, Prokocimer P. 17 January 2016. Characterization of the haematological profile of 21 days of tedizolid in healthy subjects. J Antimicrob Chemother. Epub ahead of print.

13. Clinical and Laboratory Standards Institute. 2015. Performance standards for antimicrobial susceptibility testing; 25 th informational supplement. M100-S25. Clinical and Laboratory Standards Institute, Wayne, PA.

14. Schmidt-Malan SM, Greenwood Quaintance KE, Karau MJ, Patel R. 2016. In vitro activity of tedizolid against staphylococci isolated from prosthetic joint infections. Diagn Microbiol Infect Dis 85:77-79. http://dx .doi.org/10.1016/j.diagmicrobio.2016.01.008.

15. Vergidis P, Rouse MS, Euba G, Karau MJ, Schmidt SM, Mandrekar JN, Steckelberg JM, Patel R. 2011. Treatment with linezolid or vancomycin in combination with rifampin is effective in an animal model of methicillin-resistant Staphylococcus aureus foreign body osteomyelitis. Antimicrob Agents Chemother 55:1182-1186. http://dx.doi.org/10 .1128/AAC.00740-10.

16. Rouse MS, Piper KE, Jacobson M, Jacofsky DJ, Steckelberg JM, Patel R. 2006. Daptomycin treatment of Staphylococcus aureus experimental chronic osteomyelitis. J Antimicrob Chemother 57:301-305. http://dx.doi .org/10.1093/jac/dki435.

17. Cubist Pharmaceuticals. 2014. Sivextro package insert. Cubist Pharmaceuticals, Lexington, MA.

18. Varoglu M, Nguyen L, Shim H, Kim E, Flanagan S. 2015. Cross-species plasma protein binding of tedizolid, abstr EV-0064. Abstr 25th Eur Congr Clin Microbiol Infect Dis.

19. Flanagan S, Passarell J, Lu Q, Fiedler-Kelly J, Ludwig E, Prokocimer P. 2014. Tedizolid population pharmacokinetics, exposure response, and target attainment. Antimicrob Agents Chemother 58:6462-6470. http: //dx.doi.org/10.1128/AAC.03423-14.

20. Pea F, Viale P, Cojutti P, Del Pin B, Zamparini E, Furlanut M. 2012. Therapeutic drug monitoring may improve safety outcomes of long-term treatment with linezolid in adult patients. J Antimicrob Chemother 67: 2034-2042. http://dx.doi.org/10.1093/jac/dks153.

21. FDA. 2014. Sivextro (tedizolid phosphate) tablets and injection. FDA briefing document. Anti-Infective Drug Advisory Committee Meeting. FDA, Rockville, MD.

22. Flanagan S, McKee EE, Das D, Tulkens PM, Hosako H, Fiedler-Kelly J, Passarell J, Radovsky A, Prokocimer P. 2015. Nonclinical and pharmacokinetic assessments to evaluate the potential of tedizolid and linezolid to affect mitochondrial function. Antimicrob Agents Chemother 59:178185. http://dx.doi.org/10.1128/AAC.03684-14.

23. Euba G, Rouse MS, Del Pozo JL, Jacobson MJ, Steckelberg JM, Patel R. 2009. Linezolid treatment of Staphylococcus aureus experimental foreign body osteomyelitis, abstr B-1322. Abstr 49th Intersci Conf Antimicrob Agents Chemother. American Society for Microbiology, Washington, DC.

24. Locke JB, Zurenko GE, Shaw KJ, Bartizal K. 2014. Tedizolid for the management of human infections: in vitro characteristics. Clin Infect Dis 58(Suppl 1):S35-S42. http://dx.doi.org/10.1093/cid/cit616.

25. Chan LC, Basuino L, Dip EC, Chambers HF. 2015. Comparative efficacies of tedizolid phosphate, vancomycin, and daptomycin in a rabbit model of methicillin-resistant Staphylococcus aureus endocarditis. Antimicrob Agents Chemother 59:3252-3256. http://dx.doi.org/10.1128/AAC .04376-14.

26. Raad I, Hanna H, Jiang Y, Dvorak T, Reitzel R, Chaiban G, Sherertz R, Hachem R. 2007. Comparative activities of daptomycin, linezolid, and tigecycline against catheter-related methicillin-resistant Staphylococcus bacteremic isolates embedded in biofilm. Antimicrob Agents Chemother 51:1656-1660. http://dx.doi.org/10.1128/AAC.00350-06. 